\section{DIVISION G COMMISSION 27}

\author{
PRESIDENT \\ VICE-PRESIDENT \\ PAST PRESIDENT \\ ORGANIZING COMMITTEE
}

\author{
VARIABLE STARS \\ ÉTOILES VARIABLES
}

\title{
TRIENNIAL REPORT (2012-2015): THE LEGACY ISSUE
}

\section{Variable Stars and Commission 27}

The study of variable stars has played a central role in astronomy for over 400 years, and more so in the present than at any time in history. Stars, especially variable stars, are astrophysical laboratories for understanding physical processes in the universe. Stars represent the fundamental components of stellar systems, galaxies and the universe.

The purpose and mission of Commission 27 (C27), facilitated by its organising committee, has been: to study and understand variable stars, both observationally and theoretically; to support and enhance scientific activity and scientific exchange by organising and/or supporting conferences, workshops and other activities which are accessible to the diverse interests and wide geographical distribution of our members; to disseminate information to the members of C27, our Division and the entire IAU community; to implement specific actions pertaining to variable star astronomy as required and resourced; and to provide a balanced and widely accessible organisation through having representatives from a wide geographical spread and gender balance.

With around 600 members listed during the 2012-2015 triennium, C27 has been a large and active commission in numerous areas of variable star research. The commission has organised successful approximately-biennial symposia, has provided web resources linking organisations active in the field, and co-sponsored (with Commission 42) the Information Bulletin on Variable Stars. C27 has strong ties with a number of other commissions concerned with stellar astrophysics, including Commission 42 (Close binary stars), and with Working Groups on Massive Stars and Supernovae, Active B-type stars, and Ap and related stars.

C27 members are interested in the observational and theoretical study of stars with variability due to a multitude of physical processes, both intrinsic and extrinsic, including: pulsation, radial and non-radial; rotation; binarity; eclipses and transits; outbursts; supernovae and explosions; magnetic phenomena; the presence of circumstellar matter, in disks and shells; and transient and long-term phenomena in all evolutionary stages of stellar lifetimes. 
The motivation for studying such phenomena is manifold. The understanding of the structure and evolution of stars is fundamental to our understanding of the universe as a whole. The exploitation of variable star phenomena is used to advance our knowledge of fundamental physical processes. This is because stars, especially variable stars, are probes for revealing key properties such as distances, physical structures and chemical evolution in stellar systems at all scales up to the cosmological.

In recent years, variable star research has been revolutionised by technological advances in ground and space-based observations and multi-wavelength instrumentation, advances in our understanding of complex physical processes through theoretical modelling, and the enormous advances in computational ability for both observational and theoretical applications. This has resulted in a rapid advancement of understanding in nearly all areas of variable star research.

Commission 27 has supported cross-divisional and cross-commission information exchange, discussion and research by recognising that our understanding of physical processes in stars contributes significantly to related areas of astrophysical research, including, but not limited to:

- the Sun as a star;

- stars as hosts of planets and planetary systems;

- stars as universal laboratories for fundamental astrophysics;

- stars as fundamental components of galaxies;

- stars as probes of chemical evolution of galaxies;

- stars as key distance indicators for the distance scale of the universe.

Of particular interest is the role of stellar pulsation as a key type of stellar variability, which is an increasingly important tool of stellar physics. The advances in observational technology, and the resulting precision and abundance of data, is making an immediate and direct impact on our science. It has advanced the study of small-amplitude solarlike oscillations in main-sequence stars and giants, discovered new classes of pulsating variable, and provided insight into the internal structure of stars across the HertzsprungRussell diagram. As a consequence, we can explore the microphysics of stars in ways hitherto impossible.

\subsection{Conferences}

The stellar pulsation community, has held (almost) biennial conferences on stellar pulsation (the "Los Alamos" series) which have been actively supported by C27 since 1971. These have been held at: 1971 Los Alamos, USA; 1974 Goddard Space Flight Center, USA; 1976 Los Alamos, USA; 1978 GSFC, USA; 1980 Los Alamos, USA; 1982 Boulder, USA; 1984 Toronto, Canada; 1986 Los Alamos, USA; 1988 University of Nebraska, USA; 1990 Bologna, Italy; 1992 Victoria, Canada; 1995 Cape Town, South Africa; 1997 Los Alamos, USA; 1999 Budapest, Hungary; 2001 Leuven, Belgium; 2003 Christchurch, New Zealand; 2005 Rome, Italy; 2007 Vancouver, Canada; 2009 Santa Fe, USA; 2011 Granada, Spain; and 2013 Wroclaw, Poland. The next meeting in the series is planned for December 2016 in San Pedro de Atacama, Chile.

Other IAU sponsored symposia and meetings that have been relevant to our membership in the most recent triennium have been:

\section{IAU Symposia:}

- IAUS 307: New Windows on Massive Stars, Asteroseismology, Interferometry and Spectropolarimetry 23-27 June 2014, Geneva, Switzerland

- IAUS 301: Precision Asteroseismology, 19-23 Aug 2013, Wroclaw, Poland (a celebration of the Scientific Opus of Wojtek Dziembowski). 
IAU-sponsored meeting:

- IAU FM17: Advances in Stellar Physics from Asteroseismology, 12-14 August 2015, IAU General Assembly, Honolulu, Hawaii.

\subsection{Half a century of pulsating variables stars in the HR diagram}

IAU Commission 27 has seen the discovery and classification of an enormous number of pulsating variable stars since its inauguration, a process whose pace is continually increasing as synoptic surveys become deeper, faster, wider and more sensitive. The pace of discovery is illustrated in Fig. 1 which compares the classes of variable known in 1965 with those known fifty years later. Fig. 1 also illustrates that the date when a class becomes established is ill-defined. Although individual members were known to vary as early as 1910, $\alpha$ Cygni variables were not recognized as a class until the publication of the 4th General Catalogue of Variables Stars (Kholopov et al. 1996)., Meanwhile, although discovered to vary in 1964, pulsations in the first ZZ Ceti pulsator (HL Tau 76) were only finally established by Landolt in 1968. Thus roughly nine classes of variable star were recognized in the 370 years between Fabricius' discovery of variability in o Ceti and 1965. In comparison, there has been an explosion in the number and diversity of compact pulsators and other small-amplitude pulsators discovered since, with at least 12 distinct classes identified in the 25 years since 1990 .

\section{Science Highlights of the Last Decade, including the 2012-2015 Triennium}

\subsection{Cepheids and RR Lyrae variables}

For almost a century, the Cepheids and RR Lyrae stars have been used as standard candles on the first rung of the cosmic distance scale. But despite their status as cosmic lighthouses, there are several long-standing and newly-apparent mysteries surrounding these stars. Cepheids are giant stars that have evolved off the main sequence into the Cepheid instability strip. They are regular radial-pulsating stars, with a well-defined period-luminosity relationship (Leavitt Law - as was discovered by Henrietta Leavitt), which makes them ideal stars to be used as primary distance indicating standard candles. The Cepheid family consists of different variable classes: Type I, Type II and anomalous Cepheids. Type I, or classical Cepheid stars, are young, bright supergiants with masses higher than 2-3 solar mass. Because of their intrinsic brightness, they can be seen at very large distances, galaxies in the Local Group and beyond, and thus they are a means by which the Hubble constant can be established. But they are not as common as their older, low-mass, less luminous counterparts, the RR Lyrae stars.

RR Lyrae stars are evolved low-mass (0.5-0.7 solar mass) stars that show pulsations with periods of typically $0.3-0.8$ days and large light variations around one magnitude (Smith 1995) . In the Hertzsprung-Russell diagram, they are located on the intersection of the horizontal branch with the (Cepheid) instability strip. The RR Lyrae stars are older and less luminous than the classical Cepheids. Thanks to their well-defined absolute magnitude, they also play a crucial role as distance indicators and Galactic structure tracers (Sesar et al. 2013). As primarily radially pulsating stars, just like the Cepheids, they are also touchstones for the modelling of stellar pulsations. Depending on their main radial eigenmode(s) and ensuing light curve shape, the RR Lyraes are divided into subclasses: fundamental-mode pulsators (F): RRab; first overtone (O1): RRc, both 

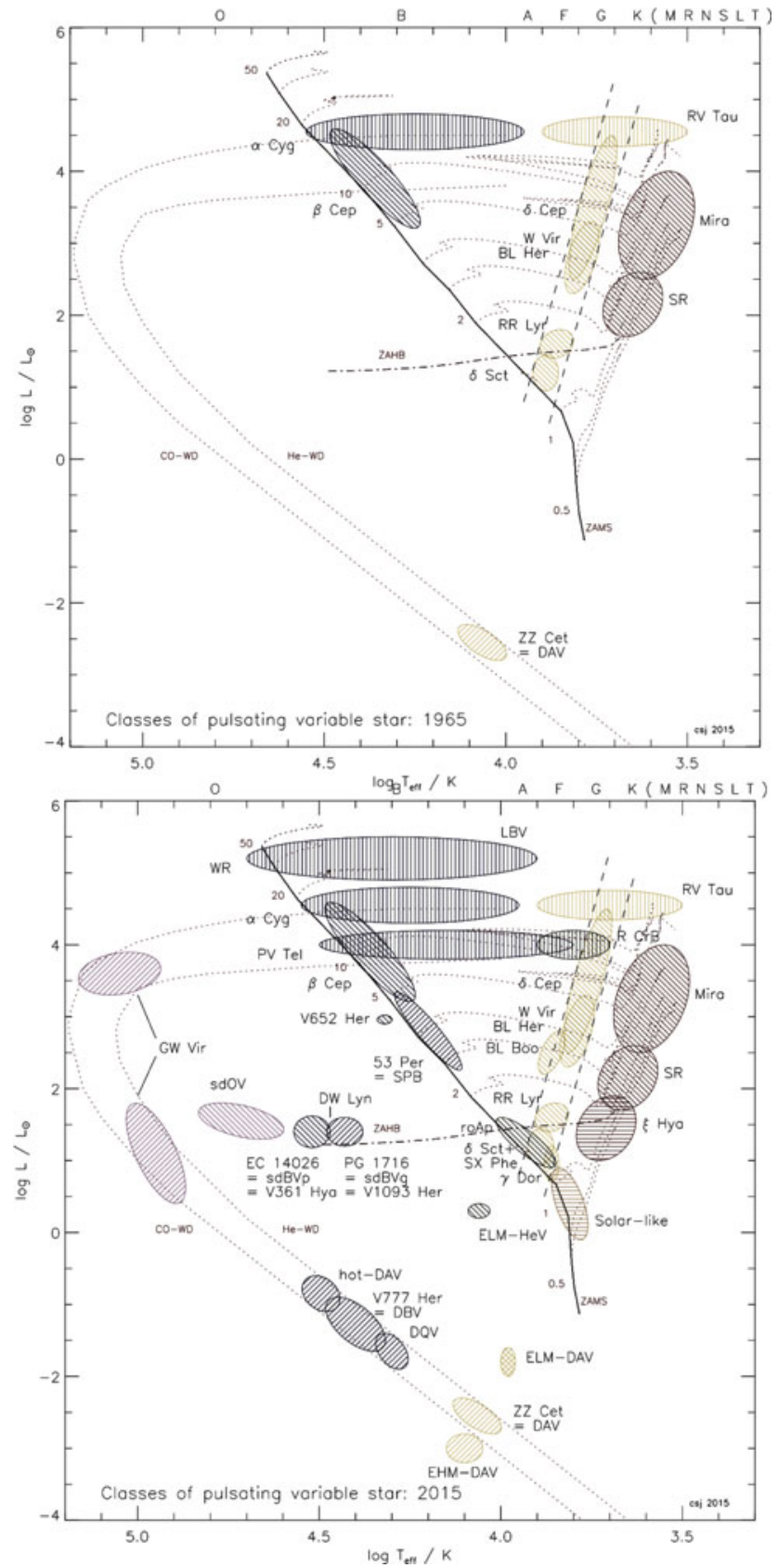

Figure 1. Luminosity-effective temperature (or Hertzsprung-Russell) diagrams showing the approximate locations of classes of pulsating variable star coloured roughly by spectral type, the zero-age main sequence and horizontal branch, the Cepheid instability strip, and evolution tracks for model stars of various masses, indicated by small numbers $\left(\mathrm{M}_{\odot}\right)$. Shadings represent opacity-driven p-modes $(\backslash \backslash \backslash)$, g-modes $(/ / /)$ and strange modes $(|| \mid)$ and acoustically-driven modes (三). Approximate spectral types are indicated on the top axis. The upper panel shows the classes which were known or identified in 1965, the lower panel shows the classes known in 2015. Based on figures by J. Christensen-Dalsgaard and subsequently by Jeffery (2008). 
$(\mathrm{F}+\mathrm{O} 1)$ : RRd. In addition to their importance in Galactic structure studies and nearfield cosmology, these stars show one of the longest-standing riddles of pulsation theory. At least half of the RRab stars (and some RRc and RRd too) exhibit the Blazhko-effect (Blazhko 1907), a periodic modulation of the amplitude and phase of the pulsation. For more than a century, its cause has been a mystery.

In the course of the past decade, survey and space data as well as dedicated campaigns of the RR Lyrae stars and Cepheids have led to a giant leap forward in our understanding of their pulsations, and along with theoretical models, as well as their structure and evolution.

RR Lyrae-focused campaigns (such as the Konkoly RR Lyrae survey - Jurcsik et al. 2009 and the Blazhko project - Kolenberg et al. 2006) provided data of selected targets, allowing in-depth studies of RR Lyrae pulsation characteristics. On the other hand, large-scale surveys such as MACHO (Alcock et al. 2003) and OGLE (Olech et al. 1999) yielded observations of many variable stars including numerous RR Lyraes as a byproduct, giving us occurrence rates of different types of RR Lyrae pulsators. With these data the gap between theoretical understanding and observations became more obvious, notably for the Blazhko effect.

The main breakthroughs for RR Lyrae stars came with space missions such as MOST (Gruberbauer et al. 2007), CoRoT (Szabo et al. 2014), and especially Kepler (Kolenberg et al. 2010, and references below). The Kepler (planet-hunting) spacecraft delivered ultra-precise, continuous photometric time series data of the $\sim 50 \mathrm{RR}$ Lyrae known in the Kepler field and one Cepheid. These data revealed an amazing variety of pulsational behaviours: previously unseen dynamical phenomena, such as period doubling (Kolenberg et al. 2010, 2011, Szabo et al. 2010), resonances (Kolláth et al. 2011), additional frequencies (Benkö et al. 2010, Nemec et al. 2011, Kolenberg et al. 2011, Guggenberger et al. 2012) often with interesting, recurring frequency ratios (Moskalik et al. 2015), and the diversity of the Blazhko modulation (including multiple modulation periods) (Benkö et al. 2014). These findings led to valuable new insights, including a new explanation for the Blazhko effect (Buchler \& Kolláth 2011). But with more insight and better data come more questions. For example: period doubling and the Blazhko effect seem to be linked (through higher order resonance?) How does period doubling depend on the physical parameters (metallicity, temperature, Galactic population, etc.)? What is the nature of the mysterious, newly found modes with frequency ratios of $\sim 0.62$ seen in RRc (and RRd) stars? (Moskalik et al. 2015). The one Cepheid observed with Kepler shows period jitter (Derekas et al. 2012) - is this a common phenomenon?

To answer all these questions, we will study high-quality data of a much larger sample of stars. K2, the resurrection of the Kepler mission after a second of its reaction wheels broke down, uses only two reaction wheels and hence it is limited to pointing near the ecliptic plane, and sequentially observes fields as it orbits the Sun. K2 allows us to significantly increase the number of high-precision RR Lyrae and Cepheid light curves, stemming from different environments (disk, bulge, halo, galaxy mergers and streams).

PLATO (Rauer et al. 2014) will have comparable capabilities, but only ten years from now. TESS (Ricker at al. 2014, launch foreseen in 2017) will have shorter runs, observe brighter targets and will have higher contamination due to its much larger pixels, not ideal to observe faint targets. The ambitious Gaia mission will provide unprecedented positional and radial velocity measurements with the accuracies needed to produce a stereoscopic and kinematic census of about one billion stars in our Galaxy and throughout the Local Group. Gaia will observe tens of thousands of RR Lyrae stars (Eyer \& Cuypers 2000). 
There are a lot of unknowns regarding the RR Lyraes progenitors, their evolution (e.g., how much mass loss occurred before they reached the horizontal branch?) and their exact physical parameters. Interestingly, out of tens of thousands of known RR Lyrae stars, less than a handful are known/suspected to be in a binary system (Wade et al. 1999). Other open questions include the role of convection in these stars, its interaction with the pulsation and its potential role in the mysterious modulation seen in many RR Lyrae stars.

To summarize: the field of RR Lyrae and Cepheid studies is vigorous and has recently gained new momentum through high-precision observations that shed new light on the dynamical phenomena inside these stars, leading to novel ideas and hypotheses. On the theoretical front multidimensional hydrodynamical codes are about to mature, allowing for an improved description of the interaction between convection and pulsation (Muthsam et al. 2010; Mundprecht et al. 2015), and the exploration of nonradial motions (Geroux \& Deupree 2015; Stellingwerf et al. 2013). Ongoing and future sky surveys, like OGLE, VVV, and LSST will (continue to) provide an overwhelming amount of data enabling to address a multitude of astrophysical questions where RR Lyrae stars play a significant role. Long-awaited astrometric data from the GAIA satellite will give a boost to the study and application of these galactic and extragalactic standard candles. The foreseen future collaboration of theorists and observational astronomers will these fascinating objects into a wide astrophysical context from pulsation theory to near-field cosmology.

\section{Cepheids}

Cepheids stars have greatly benefited from recent theoretical and observational developments. Here we list a few (non exhaustive) striking achievements:

(1) The introduction of rotation in stellar evolution models gives a clue for solving the Cepheid mass discrepancy without invoking overshooting or mass loss (Anderson et al. 2014).

(2) OGLE is continuing its remarkable harvest (e.g. Soszyński et al. 2010, Soszyński et al. 2011, Soszyński et al. 2015). Thanks to the detailed light curves from OGLE photometry, classical Cepheids were surprisingly found behind the bulge, above the Galactic plane by Feast et al. (2014).

(3) Radius variations of Cepheids are nearly routinely measured with interferometry, providing with other observables (radial velocity and photometry) distances to the stellar objects (Mérand et al. 2015). When a star distance is known, the method can be inverted and the debated factor which converts radial velocity measurements into the change of radius due to pulsation, the p-factor, can be studied. The p-factor seems to have only a weak dependence on the period (Breitfelder 2015).

(4) The pulsation of Cepheids may not be so regular: cycle to cycle variations have been detected with the ever improving precision of radial velocity measurement (Anderson et al. 2014).

(5) On the side of astrometry, Gaia will provide exquisite parallaxes in addition to new Cepheid discoveries. Also from space, an HST program is achieving remarkable results in the distance determination up to $5 \mathrm{kpc}$ (Riess et al. 2014).

In conclusion, within a few years, all these developments have made the research field on Cepheids very lively and interesting.

\subsection{Giant Pulsators}

Like the Sun, red giants show surface convection, which stochastically excites standing sound waves. While measurements of these oscillations had been limited in the past due 
to the low amplitude of the oscillations, the past decade saw the field accelerate, mainly driven by space missions, including MOST, WIRE, CoRoT and Kepler.

Before asteroseismology entered the high-precision photometry space age, observations were carried out from ground, and most successfully using spectroscopy. The early 1990s saw the first attempts to measure solar-like oscillations in giants, which all used photometry (e.g. Gilliland et al. 1993 and references therein), but it took a decade before the first firm detection was achieved (Frandsen et al. 2002) thanks to newly developed high-precision spectrographs built to find exoplanets. Even after detections of oscillations in about a dozen stars from both ground (Barban et al. 2004; De Ridder et al. 2006) and early space missions (Buzasi et al. 2000, Retter et al. 2003, Barban et al. 2007, Gilliland 2008), it was not clear which oscillations modes where present and how useful they would be for seismic inferences. A ten-telescope strong campaign aimed at the red giants in the open cluster M67 resulted in only marginal detections, which concluded almost two decades of ground-based photometric attempts (Stello et al. 2007).

It was clearly photometric data from space missions that transformed the field into something useful for other fields of astrophysics. A sample of hundreds of stars observed by CoRoT unambiguously demonstrated that red giants exhibit both radial and nonradial modes (De Ridder et al. 2009). Subsequently, Kepler data revealed that the red giant frequency spectra were rich in information, including that of the core, which for exampled enabled the distinction to be made between red giant branch stars and red clump stars. Kepler results also provided measurement of radial differential rotation in red giants (Beck et al. 2012, Mosser et al. 2012a), which strongly indicated that current modelling of stellar evolution is lacking physical mechanisms that are efficient in transporting angular momentum from the core to the envelope as stars evolve from the main sequence to the red giant phase. Puzzles about mode identification raised by decadelong observations of very luminous M giants (semi-regulars) from ground (Soszyński, Wood \& Udalski 2013) Kepler data (Mosser et al. 2013) as a natural extrapolation of the solar-like oscillation known for less evolved stars (Stello et al. 2014).

However, one interesting puzzle arising from the Kepler data was the presence of a few dozen red giants with almost no dipole modes (Mosser et al. 2012b). Recent result show strong indications this effect is due to strong magnetic fields inside the stars - a result that could seed a new line of research in the next few years. The shear numbers of red giants with detected oscillations has risen by orders of magnitude in a period of only $2-3$ years, to roughly 15-20 thousand stars (Mosser et al. 2010, Kallinger et al. 2010, Hekker et al. 2011, Huber et al. 2011, Miglio et al. 2012, Stello et al. 2013). With these large numbers of stars we can expect to resolve new questions and opened up completely new ways of exploring red giants using asteroseismology in the decade to come.

\subsection{Solar-like variables}

In stars with convective outer layers, global oscillations can be excited by turbulent motions. In this process the energy of the convective noise is effectively transformed into global oscillation modes that are stochastically excited and damped. The Sun exhibits such stochastic oscillations and these are expected to be excited in all stars with convective outer layers, such as low-mass main-sequence stars, subgiants and red-giant stars.

Over the past 10-15 years the study of solar-like variables has made tremendous progress, with first confirmations of such oscillations in stars other then the Sun from ground-based spectroscopy for $\eta$ Bootis (G0IV, Kjeldsen et al. 2003), $\alpha$ Cen A (G2V, Butler et al. 2004) and B (K1V, Carrier \& Bourban 2003), Procyon (F5IV, Eggenberger et al. 2004) and $\xi$ Hydrae (G7III, Frandsen et al. 2002). Ground-based multi-side spectroscopic campaigns such as the ones for $\epsilon$ Ophiuchi (G9.5III, De Ridder et al. 2006) 
with two telescopes and Procyon (Arentoft et al. 2008) with eleven telescopes were the state-of-the-art up to the photometric space revolution of the CoRoT and Kepler missions.

CoRoT observed a few tens of solar-like oscillators with the seismic channel. For HD 49933 (F3V) cyclic frequency variations associated with the presence of a stellar magnetic-activity cycle in a star other than the Sun were detected (Garcia et al. 2010). Additionally, Miglio et al. (2010) provided first evidence of a sharp internal structure change in the variations of the frequencies of HR 7349 (G8III, Carrier et al. 2010).

In addition to tens of targets observed with the seismic channel, tens of thousands of stars, among which many red-giant stars, were observed with the exoplanet channel (Hekker et al. 2009). Such large datasets in different parts of the Milky Way complemented with (spectroscopic) temperatures and chemical abundances were recognised to be of interest for population studies (Miglio et al. 2009).

With data from the Kepler spacecraft the revolution for solar-like variables continued with detections of oscillations in hundreds of dwarfs and subgiants (Chaplin et al. 2011) as well as thousands of red-giant stars (Hekker et al. 2011; Stello et al. 2013). For the giant stars mixed-modes have been detected (Beck et al. 2011; Bedding et al. 2011) which make it possible to study the inner parts of these stars in a more direct manner.

In the past triennium the long timeseries data of the Kepler missions have been further explored. As a result of the high frequency resolution, rotational splittings of the oscillation modes could be detected. This revealed radial differential rotation in subgiants (Deheuvels et al. 2012, 2014), early red-giants (Beck et al. 2012) and red giants in later phases (Mosser et al. 2012a) with the cores of these stars rotating faster then the surface, while the core rotation significantly slows down during the red-giant phase. From the rotational splitting the inclination angle of the spin axis of the star can also be derived. For planet-host stars with eclipsing planets this has led to the detection of spinorbit (mis)alignments (Chaplin et al. 2013; Huber et al. 2013). These (mis)alignments provide vital information regarding the formation and evolution of planetary systems. Other highlights are, among others, the detailed analysis of the detections of individual frequencies in tens of main-sequence stars (Appourchaux et al. 2012), and the classification of evolved stars from their mixed-mode pattern (Mosser et al. 2014). Additionally, work on testing and improving our current stellar models has started with the inclusion of different descriptions of rotation (Cantiello et al. 2014) and mixing in Helium core burning stars (Constantino et al. 2015).

\subsection{Main sequence $A-F$ stars}

Our understanding of the oscillation spectra in the A-F stars stars has improved thanks to the Kepler survey which revealed thousands of new variable stars. Results of investigation of A stars from the Kepler field-of-view shows that at least 56 per cent of A-F stars in the $\delta$ Sct instability strip pulsate at amplitudes above $50 \mu \mathrm{mag}$ (Murphy 2014) and have shown that the presence of low frequencies and hybrid pulsations is a property of all $\delta$ Scuti stars (Balona et al. 2015a). Balona et al. (2013a) found that about 40 per cent of A stars show differential rotation typical to Sun; surprisingly A stars have starspot variability and are active like a cooler stars, and about 1.5 per cent show flares.

Bradley et al. (2015) reported results of studies of 2768 Kepler stars, among them 207 $\gamma$ Dor, $84 \delta$ Scuti and 32 hybrid candidate stars. Their discoveries, coupled with the work of others, show that Kepler discovered over $2000 \gamma$ Dor, $\delta$ Sct and hybrid star candidates in the Kepler field of view.

Murphy et al. (2014), applied a method for finding binaries that yields the full radial velocity curves from the Kepler photometry of pulsating stars and found several 
multi-mode $\delta$ Scuti type stars, including a system KIC 4471379 in which both components are pulsating. Keen et al. (2015) examined another spectroscopic binary system, KIC 10080943, in which both components appear to be $\gamma$ Dor- $\delta$ Sct pulsators.

Kurtz et al. (2015) demonstrated the validity of the frequency modulation technique using the Kepler photometric data of KIC 8569819 - the eccentric spectroscopic binary consists of an F-type hybrid pulsator and a G-type companion star.

Bognar et al. (2015) detected $\delta$ Sct- $\gamma$ Dor type oscillations in the unique multiple system KIC 9533489, which consists of a pulsating F0 star, a fainter star at 1", an unseen G/K star (either gravitationally bound or unbound) and an $\mathrm{R}=1.6 R_{\mathrm{jup}}$ exoplanet orbiting the $\mathrm{G} / \mathrm{K}$ star.

Ripepi et al. (2015) found six pre-main sequence and one $\gamma$ Dor-type pulsating stars in the analysis of 27 Kepler variable stars.

Lehmann et al. (2015), from the analysis of extended spectroscopic time series of the $\delta$ Scuti-type WASP-33b exoplanet host star, detected orbital and pulsational radial velocity variations, and gave a new estimation of the exoplanet mass as $2.1 \pm 0.2$ Jupiter masses.

Neiner \& Lampens (2015) reported the first discovery of a weak ( $\sim 76 G)$ magnetic field in a main-sequence Kepler $\delta$ Sct- $\gamma$ Dor hybrid pulsator HD 188774.

Spectroscopic analysis of 56 chemical elements in pulsating $\delta$ Sct stars $\rho$ Pup and $\delta$ Sct itself (Yushchenko et al. 2015) revealed a correlation between relative abundances of elements versus the second ionization potentials, having discontinuities at 13.6 and $24.6 \mathrm{eV}$ - the ionization potentials of hydrogen and helium, respectively. These correlations have been explained as accretion of interstellar gas onto the stellar atmosphere.

Holdsworth et al. (2014a) have searched for short-period pulsators with pulsation amplitudes greater than 0.5 millimagnitude over 1.5 million targets from the WASP survey. They reveal over 350 stars which pulsate with periods less than 30 minutes; among them 10 new roAp, 13 pulsating Am stars and the fastest known pulsating $\delta$ Scuti stars.

Holdsworth et al. (2014b) reported the safe detection in the Kepler and WASP data sets of pulsation frequency variations in the roAp star KIC 7582608. They concluded that one possibility for the frequency variation is intrinsic variability in the pulsation cavity or the binary motion.

Kepler photometry revealed roAp stars that possessed low frequency variations of unknown driving mechanism, in KIC 8677585 (Balona et. al. 2013a), KIC 10483436 and KIC 10195926 (Balona et al., 2013b). Smalley et al. (2015) reported the detection in KIC 4768731 of approximate 23-minute oscillations, but with a period that was variable over the 4-year time span.

Hartmann \& Hatzes (2015) carried out a spectroscopic survey of 65 chemically-peculiar stars using HARPS at the 3.6-m telescope at ESO. They discovered spectroscopic binary Ap stars and, among them, the roAp star HD 42659 with 93.2-day radial velocity variations.

\subsection{B-star pulsations}

It is important to remember that the exact mechanism exciting pulsations in B-type stars was discovered only some 20 years ago (Moskalik \& Dziembowski 1992). It was quickly realized heavy-element opacities play a crucial role in those stars, and can be constrained by analysing the pulsations asteroseismically. In this regard, an important step was the discovery of $\beta$ Cephei stars in the LMC (e.g. Kołaczkowski et al. 2004). At about the same time, large observing campaigns were organized for a number of selected stars that led to first determinations of the interior structure of some stars, including constraints on convective overshooting and differential interior rotation (e.g. Aerts et al. 
2003, Pamyatnykh et al. 2004). Thanks to analyses of large photometric surveys, over 200 galactic $\beta$ Cephei stars are nowadays known (Pigulski \& Pojmański 2008), several of them also showing SPB-type oscillations, i.e. they are "hybrid" pulsators, having the potential of even more in-depth seismic information to be obtained (Handler et al. 2009).

The latest years of B-star pulsation research have been dominated by studies from space, due to superior data quality. Saio et al. (2006) reported the first case of a pulsating B-type supergiant, which can be explained by state-of-the-art models (Saio et al. 2013, Ostrowski \& Daszyńska-Daszkiewicz 2015). For main-sequence pulsators, large numbers of oscillation modes were detected from space (e.g. Degroote et al. 2010, Pápics et al. 2012), including perhaps even oscillations stochastically excited by sub-surface convection (Cantiello et al. 2009, Belkacem et al. 2010). Variability in B stars is however not restricted to pulsation only. This is particularly present in Be stars, where outbursts, rotational modulation and accretion may all be present at the same time (Huat et al. 2009). An excellent recent overview of the latest developments in the field of B-star pulsations has been provided by Aerts (2015).

It is to be expected that research on B-star variability will continue to flourish. New and upcoming space missions, like BRITE-Constellation (Weiss et al. 2014) whose primary science target are just those stars and TESS (Ricker et al. 2014), performing an all-sky variability survey of bright stars, do and will deliver exclusive data, with better prospects for ground-based support. At the same time, theory is maturing (e.g. Paxton et al. 2015), leaving good reasons for looking optimistically into the future.

\subsection{Compact oscillators}

For compact pulsators, the Sloan Digital Sky Survey has been the tool to find and characterize new white dwarf and subdwarf stars, and led to the discovery of more than 200 new pulsators, mainly DAVs, also called ZZ Cetis (Mukadam et al. 2004; Mullally et al. 2005; Kepler et al. 2005a; Castanheira et al. 2006, 2007), and their modelling (Castanheira \& Kepler 2008, 2009; Romero et al. 2012, 2013), but also DBVs Nitta et al. (2009) and a few DOVs, also called variable PG 1159 or GW Vir stars (Quirion 2009a; Quirion, Fontaine \& Brassard 2009b; Woudt, Warner,\& Zietsman 2012; Kepler et al. 2014). Two new classes of white dwarf pulsators were found, the DQVs (Montgomery et al. 2008; Fontaine et al. 2009; Williams et al. 2013) and the ELMVs Hermes et al. (2013a,b); Van Grootel et al. (2012, 2013a); Córsico \& Althaus (2014).

The Kepler satellite has been essential to observe a handful of bright compact pulsators (Hermes et al. 2014; Bell et al. 2015; Hermes et al. 2015).

Reviews of pulsating white dwarfs have been published by Winget \& Kepler (2008), Fontaine \& Brassard (2008), and Althaus et al. (2010).

Kepler et al. (2005b) and Mukadam et al. (2013) measured the rate of evolution of $T_{\text {eff }} \simeq 12000 \mathrm{~K}$ white dwarfs using the rate of change of their pulsation periods, while Costa et al. (2008a) measured $\dot{P}$ for a $T_{\text {eff }} \simeq 140000 \mathrm{~K}$, PG $1159-035$ pulsator. Costa \& Kepler (2008b) and Charpinet, Fontaine, \& Brassard (2009) used multiple pulsation periods to study the internal rotation profiles of white dwarfs.

Pulsations are found in hot subdwarfs in the range $T_{\text {eff }} \simeq 20000-40000 \mathrm{~K}$ and $\log g \simeq 5.0-6.2(\mathrm{cgs})$. They belong to two groups of nonradial pulsators. The first group, named the V361 Hya (originally EC 14026) stars (Kilkenny et al. 1997), exhibits pulsation periods in the range $80-600 \mathrm{~s}$ with amplitudes of a few milli-magnitudes. The pulsations correspond to low-order low-degree p-modes, which, in sdB stars, have significant amplitudes mainly in the outermost layers (Charpinet et al. 2000). The second group, discovered by (Green et al. 2003) and called V1093 Her stars, exhibits periods from 45 min to a few hours (Van Grootel et al. 2013b). The first He-sdBV pulsator was 
discovered by Ahmad \& Jeffery (2005) and the first sdOV by Woudt et al. (2006). The Kepler satellite has had a transformational impact on the asteroseismology of V1093 Her stars, providing rotation diagnostics and, with time variable multiplet spacings, challenging conventional models of sdB interiors (e.g. Reed \& Foster 2014).

A recent review on subdwarf pulsators was published by Randall et al. (2014).

\section{The Future: IAU Commission G4 on Pulsating Stars}

Following the 2012 decision to reform the Division and Commission structure of the IAU, the Organizing Committee of Commission 27 acknowledged that in recent years the major activities of the Commission had substantially shifted towards work on pulsating stars and asteroseismology. Consequently, in 2014, a proposal was submitted for a reconstituted Commission on Pulsating Stars, since these represent a key type of stellar variability, which is playing an increasingly important role in the study of stellar physics. Following review and a popular vote, the proposal was approved by the IAU reform committee, and Commission G4 'Pulsating Stars' was officially constituted at the XXIXth IAU General Assembly in 2015.

Commission G4 (CG4) commences work in an era when the Kepler and CoRoT projects, together with ground-based synoptic surveys, have precipitated a revolution in the measurement of the absolute dimensions and internal properties of stars of all types. New discoveries have enabled the tools of asteroseismology to be applied to stars with diverse internal structures and physics, furthering our understanding of how stars work, providing calibrations for cosmological standard candles and characterising exoplanet host stars. Developing techniques and tools to exploit this new knowledge will be a cornerstone in the search for habitable Earth-like planets following the 2017 NASA launch of TESS and the 2024 ESA launch of Plato (PLanetary Transits and Oscillations of stars), and for exoplanet studies with MIRI/JWST and E-ELT, whilst our understanding of the pulsating star populations of the Galaxy will be transformed as LSST and Gaia discover many new pulsating stars and accumulate precise constraints on galactic structure and stellar dimensions.

Driven by large space missions, ground-based synoptic surveys and multi-site collaborations, pulsating-star astronomers represent one of the most rapidly expanding and active science communities in astronomy, with a young and global demographic. Like its predecessor, CG4 will continue to play a major role in promoting communication, mobility, and equality of opportunity, and in raising the profile of young astronomers at IAU meetings and elsewhere. CG4 will also encourage the increasingly important contribution of amateur astronomers to the science of pulsating stars.

In the 2015-18 triennium, the commission work plan will include:

i) Support for cross-divisional and cross-commission information exchange, discussion and research.

ii) Promotion of biennial IAU Symposia and other conferences n Stellar Pulsation. Following CoRoT, Kepler and OGLE and the commencement of new wide-field synoptic surveys (such as K2, TESS, and Plato), as well as more focussed efforts (such as SONG and BRITE), the Symposia will continue to define progress in the field.

iii) Encourage dialogue between scientific consortia, in particular between long-term ground-based surveys and high-cadence space missions.

iv) Develop a coherent body of information based around a redesigned Commission web site to promote communication amongst scientists working in the field. 
v) Encourage members to advertise skills and experience in order, for example, to assist other Commissions, WGs, meeting organisers and consortia to identify potential speakers and/or collaborators.

vi) Support the work of the International Bulletin of Variable Stars and the General Catalogue of Variable Stars.

vii) Investigate the use of social media (e.g. FaceBook, ResearchGate, LinkedIn) to promote collaboration and dissemination of relevant results.

To deliver this programme, the commission will be led by an Organizing Committee consisting of Simon Jeffery (President), Jaymie Matthews (Vice-President), Saskia Hekker (Secretary), Karen Pollard, Dennis Stello, Joyce Guzik and Hiromoto Shibahashi.

This Legacy triennial report was produced by the Organising Committee of Commission 27: Karen Pollard (President) Simon Jeffery (Vice-President), Gerald Handler (Past President), Márcio Catelan, Laurent Eyer, Saskia Hekker, Katrien Kolenberg, David Mkrtichian, S. O. Kepler, Dennis Stello, Klaus Strassmeier and Katrien Uytterhoeven.

As President of Commission 27 I wish to thank the entire OC for their active participation and enthusiasm over the past triennium. I also extend best wishes to all past members of Commission 27 - we look forward to embarking on a new and active era for research on variable stars under the revised commission and division organisational structure of the IAU.

Karen Pollard

President of President of IAU Commission 27 Variable Stars

\section{References}

Aerts, C., 2015, Proc. IAU Symp. 307, 154

Aerts, C., Thoul, A., Daszyńska, J. et al., 2003, Science 300, 1926

Ahmad A., Jeffery C. S., 2005, A\&A, 437, L51

Alcock C., Alves, D. R., Becker, A. et al., 2003, ApJ, 598, 597

Althaus L. G., Córsico A. H., Isern J., García-Berro E., 2010, A\&ARv, 18, 471

Anderson, R. I,, Ekström, S., \& Georgy, C. et al., 2014, A\&AA, 564, 100

Anderson, R. I., 2014, A\&A A, 566, 10

Appourchaux, T., Chaplin, W. J., García, R. A. et al., 2012, A\& A, 543, A54

Arentoft, T., Kjeldsen, H., Bedding, T. R. et al., 2008, ApJ, 687, 1180-1190

Balona L. A., 2013a, MNRAS, 431, 2240

Balona, L. A., 2013b, MNRAS, 436, 1415

Balona, L. A.,Catanzaro, G., Crause, L. et al., 2013, MNRAS, 432, 2808

Balona, L. A., Daszynska-Daszkiewicz, J., \& Pamyatnykh, A. A., 2015a, MNRAS, 452, 3073

Balona, L. A., Briquet, M., Bruntt, H., et al., 2015b, MNRAS, 452, 3334

Barban, C., De Ridder, J., Mazumdar, A. et al., 2004, ESA Special Publication, 559, 113

Barban, C., Matthews, J. M., De Ridder, J. et al., 2011, 469, 1033-1038

Beck, P. G. , Bedding, T. R., Mosser, B. et al., 2011, Science, 332, 205

Beck, P. G. , Montalban, J. , Kallinger, T. et al., 2012, Nature, 481, 55-57

Bedding, T. R., Mosser, B. , Huber, D. et al., 2011, Nature, 471, 608

Belkacem, K., Dupret, M. A., \& Noels, A., 2010, A\&A, 510, A6

Bell K. J., Hermes J. J., Bischoff-Kim A. et al., 2015, ApJ, 809, 14

Benkö, J. M., Kolenberg, K. et al., 2010, MNRAS, 409, 1585

Benkö, J. M., 2014, ApJS, 213, 31 
Blazhko, S., 1907, Mitteilung über veränderliche Sterne AN, 175, 325

Bognár, Zs., Lampens, P., Fremat, Y., et al., 2015, A\&A, 581, 77

Bradley, P. A., Guzik, J. A., Miles, L. F. et al., 2015, AJ, 149, 68

Breitfelder, J., 2015, PhD Thesis, Observatoire de Paris

Buchler, J. R., \& Kolláth, Z., 2011, ApJ, 731, 24

Butler, R. P. ,Bedding, T. R., Kjeldsen, H. et al., 2004, ApJ, 600, L75-78

Buzasi, D., Catanzarite, J., Laher, R. et al., ApJ, 532, L133-136

Cantiello, M., Langer, N., Brott, I. et al., 2009, A\&A, 499, 279

Cantiello, M., Mankovich, C., Bildsten, L., Christensen-Dalsgaard, J., \& Paxton, B., 2014, ApJ, 78,93

Carrier, F. , \& Bourban, G., 2003, A\&3A, 406, L23-26

Carrier, F., De Ridder, J., Baudin, F. et al., 2003, A\& $A$, 509, A73

Castanheira B. G. et al., 2006, A\&A, 450, 227

Castanheira B. G. et al., 2007, A\&A, 462, 989

Castanheira B. G., Kepler S. O., 2008, MNRAS, 385, 430

Castanheira B. G., Kepler S. O., 2009, MNRAS, 396, 1709

Chaplin, W. J., Kjeldsen, H., Christensen-Dalsgaard, J. et al., 2011, Science, 332, 213

Chaplin, W. J., Sanchis-Ojeda, R., Campante, T. L. et al., 2013, ApJ, 766, 101

Charpinet S., Fontaine G., Brassard P., Dorman B., 2000, ApJS, 131, 223

Charpinet S., Fontaine G., Brassard P., 2009, Nature, 461, 501

Constantino, T., Campbell, S. W., Christensen-Dalsgaard, J., Lattanzio, J. C., \& Stello, D., 2015, MNRAS, 452, 123-145

Córsico A. H., Althaus L. G., 2014, A\&3A, 569, A106

Costa J. E. S. et al., 2008, $A \& A, 477,627$

Costa J. E. S. \& Kepler S. O., 2008, A\&SA, 489, 1225

Degroote, P., Aerts, C., Baglin, A. et al., 2010, Nature 464, 259

Deheuvels, S., García, R. A., Chaplin, W. J., et al., 2012, ApJ, 756, 19

Deheuvels, S., Doğan, G., Goupil, M. J. et al., 2014, A\&GA, 564, A27

Derekas, A., Szabó, Gy. et al., 2012, MNRAS 425, 1312

De Ridder, J., Barban, C., Carrier, F. et al., 2006, A\&A, 448, 689-695

De Ridder, J., Barban, C., Baudin, F et al., 2009, Nature, 459 398-400

Drake A. J., Graham, M. J., \& Djorgovski, S. G., 2014, ApJS, 213, 9

Eggenberger, P. , Carrier, F. , Bouchy, F. , \& Blecha, A., 2004, A\& A, 422, 247-252

Eyer, L. \& Blake, C., 2005, MNRAS 358, 30

Eyer, L. \& Cuypers, J., 2000, ASPC 203, 71

Feast, M. W., Menzies, J. W., Matsunaga, N., \& Whitelock, P. A., 2014, Nature, 509, 342

Fontaine G., Brassard P., 2008, PASP, 120, 1043

Fontaine G., Brassard P., Dufour P., Green E. M., Liebert J., 2009, JPhCS, 172, 012066

Frandsen, S., Carrier, F. , Aerts, C. et al., 2002, A\& 4 , 394, L5-L8

García, R. A., Mathur, S., Salabert, D. et al., 2010, Science, 329, 1032

Geroux, C. M., \& Deupree, R., 2015, ApJ 800, 35

Gilliland, R. L., 2008, AJ, 136, 566-579

Gilliland, R. L., Brown, T. M., Christensen-Dalsgaard, J. et al., 2010, PASP 122, 131

Gilliland, R. L., Brown, T. M., Kjeldsen H. et al., 1993, AJ, 106, 2441-2476

Green E. M. et al., 2003, ApJ, 583, L31

Gruberbauer, M., Kolenberg, K., Rowe, J. et al., 2007, MNRAS 379, 1498

Guggenberger, E., Kolenberg, K. et al., 2012, MNRAS, 424, 649

Handler, G., Matthews, J. M., Eaton, J. A. et al., 2009, ApJ 698, L56

Hartmann, M. \& Hatzes, A. P., 2015, A\&广A, 582, 84

Hekker, S., Kallinger, T., Baudin, F. et al., 2009, A\&SA, 506, 465-469

Hekker, S., Gilliland, R. L., Elsworth, Y. et al., 2011, MNRAS, 414, 2594-2601

Hermes J. J. et al., 2013, ApJ, 765, 102

Hermes J. J. et al., 2013, MNRAS, 436, 3573

Hermes J. J. et al., 2014, ApJ, 789, 85 
Hermes J. J. et al., 2015, ApJ, 810, L5

Holdsworth, Daniel L., Smalley, B., Kurtz, D. W. et al., 2014a, MNRAS, 443, 2049

Holdsworth, D. L., Smalley, B., Gillon, M. et al., 2014b, MNRAS, 439, 2078

Huat, A.-L., Hubert, A.-M., Baudin, F. et al., 2009, A\&A 506, 95

Huber, D., Bedding, T. R., Stello, D. et al., 2011, ApJ, 743, 143

Huber, D., Carter, J. A., Barbieri, M., Miglio, A. et al., Science, 342, 331-334

Jeffery C. S., 2008, CoAst, 157, 240

Jurcsik, J., Sódor, Á., Szeidl, B. et al., 2009, MNRAS, 400, 1006

Kallinger, T., Mosser, B., \& Hekker, S., 2010, A\&A, 522, A1

Keen, M. A., Bedding, T. R., Murphy, S. J., Schmid, V. S., Aerts, C., Tkachenko, A., Ouazzani, R.-M., \& Kurtz, D. W., 2015, MNRAS, 454, 1792

Kepler S. O., Fraga L., Winget D. E. et al., 2014, MNRAS, 442, 2278

Kepler S. O., Castanheira B. G., Saraiva M. F. O. et al., 2005, A\&BA, 442, 629

Kepler S. O. et al., 2005, ApJ, 634, 1311

Kholopov P. N., Samus N. N., Frolov M. S. et al., 1996, VizieR Online Data Catalog: General Catalog of Variable Stars, 4th Ed. (GCVS4)

Kilkenny D., Koen C., O'Donoghue D., Stobie R. S., 1997, MNRAS, 285, 640

Kjeldsen, H., Bedding, T. R., Baldry, I. K. et al., AJ, 126, 1483-1488

Kołaczkowski, Z., Pigulski, A., Soszyński I. et al., 2004, ASP Conf. Ser. 310, 225

Kolenberg K., Smith, H. A., Gazeas, K. D. et al., 2006, A\& A, 459, 577

Kolenberg K., Szabó, R.; Kurtz, D. W. et al., 2010, ApJ, 713, 198

Kolenberg, K., Bryson, S., Szabó, R. et al., 2011, MNRAS 411, 878

Kolláth, Z., Molnár, L., \& Szabó, R., 2011, MNRAS 414, 1111

Kurtz, D. W., Hambleton, K. M., Shibahashi, H., Murphy, S. J., \& Prsa, A., 2015, MNRAS, 446,1223

Landolt A. U., 1968, ApJ 153, 151

Lehmann, H., Guenther, E., Sebastian, D. et al., 2015, A\& A, 578, L4

Merand, A., Kervella, P., Breitfelder, J. et al., 2015, arXiv151001940

Miglio, A., Montalbán, J., Baudin, F. et al., 2009, 503, L21-24

Miglio, A., Montalbán, J., Carrier, F. et al., A $ध A$, 520, L6

Miglio, A., Morel, T., Barbieri, M. et al., 2012, EPJ Web of Conferences, Volume 19, 5012

Molnár, L., Plachy, E., \& Szabó, R., 2014,IBVS 6108

Montgomery M. H., Williams K. A., Winget D. E. et al., 2008, ApJ, 678, L51

Moskalik, P., \& Dziembowski, W. A., 1992, A\&A, 256, L5

Moskalik, P., Smolec, R., Kolenberg, K. et al., 2015, MNRAS 447, 2348

Mosser, B., Belkacem, K., Goupil, M.-J. et al., 2010, A\&A, 517, A22

Mosser, B., Goupil, M. J., Belkacem, K. et al., 2012, A\& A, 548, A10

Mosser, B., Isworth, Y., Hekker, S. et al., 2012, A\&A, 537, A30

Mosser, B., Dziembowski, W. A., Belkacem, K. et al., A $\& A$, 559, A137

Mosser, B., Benomar, O., Belkacem, K. et al., A $\xi A, 572$, L5

Mukadam A. S. et al., 2004, ApJ, 607, 982

Mukadam A. S. et al., 2013, ApJ, 771, 17

Mullally F., Thompson S. E., Castanheira B. G. et al., 2005, ApJ, 625, 966

Mundprecht, E., Muthsam, H. J., \& Kupka, F., 2015, MNRAS arXiv:1502.00449

Murphy, S.,J., 2014, PhD Thesis, University of Central Lancashire

Muthsam, H. J., Kupka, F., Löw-Baselli, B. et al., 2010, NewAst 15

Neiner, C., \& Lampens, P.,, 2015, MNRAS, 454, L86

Nemec, J, Smolec, R. et al., 2011, MNRAS 417, 1022

Nemec, J, Cohen, J. G. et al., 2013, MNRAS, 773, 181

Nitta A. et al., 2009, ApJ, 690, 560

Olech, A., Kaluzny, J., Thompson, I. B. et al., 1999, AJ, 118, 442

Ostrowski, J., \& Daszyńska-Daszkiewicz, J., 2015, MNRAS 447, 2378

Pamyatnykh, A. A., Handler G., \& Dziembowski, W. A., 2004, MNRAS 350, 1022

Pápics, P. I., Briquet, M., Baglin, A. et al., 2012, A\&A 542, A55 
Paxton, B. Marchant, P., Schwab, J. et al., 2015, ApJS 220, 15

Pigulski, A., \& Pojmański, G., 2008, A\&A 477, 917

Quirion P.-O., 2009, CoAst, 159, 99

Quirion P.-O., Fontaine G., \& Brassard P., 2009, JPhCS, 172, 012077

Randall S. K., Fontaine G., Charpinet S., Van Grootel V., \& Brassard P., 2014, IAUS, 301, 289

Reed, M. \& Foster, H., 2014, ASPCS, 481, 45

Rauer, H., Catala, C. et al., 2014, ExpAst 38, 249

Retter, A., Bedding, T. R., Buzasi, D. L., Kjeldsen, H., \& Kiss, L. L., 2003, ApJ, 591, L151-154

Ricker, G. R., Winn, J. N., Vanderspek, R. et al., 2014, Proc. SPIE 9143, 20

Riess, A. G., Casertano, S., Anderson, J., MacKenty, J., \& Filippenko, A. V., 2014, ApJ, 785, 161

Ripepi, V., Balona, L., Catanzaro, G. et al., 2015, MNRAS, 454, 2606

Romero A. D., Córsico A. H., Althaus L. G. et al., 2012, MNRAS, 420, 1462

Romero A. D., Kepler S. O., Córsico A. H., Althaus L. G., \& Fraga L., 2013, ApJ, 779, 58

Saio, H., Kuschnig, R., Gautschy, A. et al., 2006, ApJ 650, 1111

Saio, H., Georgy, C., \& Meynet, G., 2013, MNRAS 433, 1246

Sesar, B., Ivezic, Z et al., 2013, AJ, 146, 21

Smalley, B., Niemczura, E., Murphy, S. J. et al., 2015, MNRAS, 452, 3334

Smith, H. A., 1995, RR Lyrae stars, Cambridge University Press

Smolec, R., \& Moskalik, P., 2008, AcA, 58, 193

Soszyński, I., Wood P. R., \& Udalski, A., 2013, ApJ, 779, 167

Soszyński, I., Poleski, R., Udalski, A. et al., 2010, AcA, 60, 17

Soszyński, I., \& Udalski, A., Pietrukowicz et al., 2011, AcA, 61, 285

Soszyński, I., Udalski, A., Szymaski, M. K. et al., 2015, AcA, 65, 233

Stellingwerf, R. et al., arXiv:1310.0535

Stello, D., Bruntt, H., Kjeldsen, H. et al., 2007, MNRAS, 377, 584-594

Stello, D., Huber, D., Bedding, T. R. et al., 2013, ApJ, 765, L41

Szabó, R. Kolláth, Z. et al., 2010, MNRAS, 409, 1244

Szabó, R. 2014, IAU Symp. Proc. 301, 241

Szabó, R., Benkö, J., Paparó, M. et al., 2014, A\&A 570, 100

Van Grootel V., Dupret M.-A., Fontaine G. et al., 2012, A\& A, 539, A87

Van Grootel V., Fontaine G., Brassard P., Dupret M.-A., 2013, ApJ, 762, 57

Van Grootel V., Charpinet S., Brassard P., Fontaine G., \& Green E. M., 2013, A\&A, 553, A97

Wade R., Donley J., Fried R. et al., 1999, AJ 118, 2442, 19

Williams K. A. et al., 2013, ApJ, 769, 123

Weiss, W. W., \& Ruciński, S. M., Moffat A. F. J. et al., 2014, PASP 126, 573

Winget D. E., Kepler S. O., 2008, ARAEAA, 46, 157

Woudt P. A. et al., 2006, MNRAS, 371, 1497

Woudt P. A., Warner B., Zietsman E., 2012, MNRAS, 426, 2137

Yushchenko, A. V., Gopka, V. F., Kang, Y.-W., et al., 2015, AJ, 149, 59 